\title{
A STUDY OF GAMMA SPECTRAL BREAK IN AGN
}

\author{
A. MARCOWITH, G. HENRI and G. PELLETIER \\ Laboratoire d'Astrophysique \\ Observatoire de Grenoble BP53X \\ F38 041 GRENOBLE Cedex FRANCE
}

Since its launch, CGRO has detected more than $20 \gamma$-ray emitting AGN, most of them associated with powerful, radio-loud, flat-spectrum objects, exhibiting VLBI superluminal motions. In the case of $3 \mathrm{C279}$, the huge value of the apparent luminosity $\left(\sim 10^{48} \mathrm{erg.s} \mathrm{s}^{-1}\right)$ and the variability time-scale of a few days (Hartmann et al., 1992) gives a very large compacity $\ell_{a p p} \simeq 200$, that is, the medium should be completely thick to $\gamma$-rays. This contradiction can be explained if the $\gamma$-rays originate from a relativistic jet pointing at a small angle with respect to the line of sight(Maraschi et al., 1992). However, the still large value of compacity suggests the existence of an inner, more compact region where pair production can take place efficiently (Henri et al., 1993). This supports the so-called "two-flow" model, where the superluminal motion is attributed to the expansion of a relativistic pair plasma heated by a MHD jet from an accretion disk (Sol et al., 1989). Hence we propose to interpret the spectral break observed in many objects around a few $\mathrm{MeV}$ (Lichti et al., 1993) by an opacity effect due to photon-photon absorption by pair production.

Assuming that photons of energy $\varepsilon_{1} m_{e} c^{2}$ interact only with $\mathrm{X}$ photons of energy $m_{e} c^{2} / \varepsilon_{1}$, and for a cylindrical jet of transverse radius $r(z)$, the opacity to pair production is $\tau_{\gamma \gamma}(z) \simeq \sigma_{T} \dot{n}\left(1 / \varepsilon_{1}\right) r^{2}(z) / c \varepsilon_{1}$ where $\dot{n}\left(1 / \varepsilon_{1}\right)$ is the density of photons produced per second by Inverse Compton process at energy $1 / \varepsilon_{1}$. The total luminosity can then be evaluated as $L_{\gamma} \simeq m_{e} c^{2} \int_{z_{\gamma}}^{\infty} \varepsilon_{1} \dot{n}\left(\varepsilon_{1}, z\right) S(z) d z$, where $z_{\gamma}$ is defined by $\tau_{\gamma \gamma}\left(z_{\gamma}\right)=1$. The jet can become optically thin to $\gamma$-rays only by a decrease of soft photons and/or pair flux along $z$. Then one obtains $L_{\gamma}=L_{t h i n} \varepsilon_{1}^{-\Delta \alpha}$, where $\Delta \alpha=\frac{s-1}{2}$ for a power-law distribution of electron with an index $s$ and an exponential decrease of soft photons. The break energy is equal to $511 \mathrm{keV}$ blueshifted by the Doppler factor. Pair annihilation photons can produce a bump at this break energy. A crucial prediction of the model is that variability at high energy should lag the low energy one, in contrast with optically thin models.

\section{References}

Hartmann, R. C., et al.: 1992,ApJ Lett., 385, L1

Lichti, G. G., et al.: 1993, these proceedings

Henri, G. , Pelletier, G., and Roland, J.: 1993,ApJ Lett., 404, L41

Maraschi, L., Ghisellini, G., and Celotti, A. : 1992,ApJ, 397, L5

Sol, H., Pelletier, G., and Asseo, E.: 1989,MNRAS, 237, 411

T. J.-L. Courvoisier and A. Blecha: Multi-Wavelength Continuum Emission of AGN, 347.

(C) 1994 IAU. Printed in the Netherlands. 